\title{
On meromorphic solutions of the Riccati differential equations
}

\author{
by Ran Ran Zhang and Zong Xuan Chen (Guangzhou)
}

\begin{abstract}
We investigate the growth and Borel exceptional values of meromorphic solutions of the Riccati differential equation

$$
w^{\prime}=a(z)+b(z) w+w^{2},
$$

where $a(z)$ and $b(z)$ are meromorphic functions. In particular, we correct a result of E. Hille [Ordinary Differential Equations in the Complex Domain, 1976] and get a precise estimate on the growth order of the transcendental meromorphic solution $w(z)$; and if at least one of $a(z)$ and $b(z)$ is non-constant, then we show that $w(z)$ has at most one Borel exceptional value. Furthermore, we construct numerous examples to illustrate our results.
\end{abstract}

1. Introduction and results. In this paper, we use the standard notations of Nevanlinna's value distribution theory ([4, 11]). We use $\lambda(f)$ and $\bar{\lambda}(f)$ to denote respectively the exponents of convergence of the zero sequence and of the sequence of distinct zeros of a meromorphic function $f(z) ; \lambda(1 / f)$ to denote the exponent of convergence of the pole sequence of $f(z)$; and $\sigma(f)$ to denote the order of growth of $f(z)$. We also use

$$
\sigma_{2}(f)=\limsup _{r \rightarrow \infty} \frac{\log ^{+} \log ^{+} T(r, f)}{\log r} \text { and } \lambda_{2}\left(\frac{1}{f}\right)=\limsup _{r \rightarrow \infty} \frac{\log ^{+} \log ^{+} N(r, f)}{\log r}
$$

to denote respectively the hyperorder of $f(z)$ and the hyperexponent of convergence of the pole sequence of $f(z)$.

The Malmquist-Yosida theorem (see, e.g., [6, Chapter 10]) states that if $R(z, w)$ is rational in $w$ with meromorphic coefficients, and if the differential equation $w^{\prime}=R(z, w)$ admits an admissible meromorphic solution, then this equation must be a Riccati differential equation

$$
w^{\prime}=a(z)+b(z) w+c(z) w^{2},
$$

where $a(z), b(z), c(z)$ are meromorphic functions. So the Riccati differential

2010 Mathematics Subject Classification: Primary 34A34; Secondary 34M05, 30D35.

Key words and phrases: Riccati differential equation, growth order, Borel exceptional value. 
equation (1.1) has taken a special position in algebraic differential equations. If $c(z) \not \equiv 0$, then by the transformation

$$
w=\frac{1}{c(z)} u-\frac{b(z)}{2 c(z)}-\frac{c^{\prime}(z)}{2 c(z)^{2}},
$$

(1.1) can be transformed into

$$
u^{\prime}=A(z)+u^{2}
$$

where

$$
A=a c-\frac{b^{2}}{4}+\frac{b^{\prime}}{2}-\frac{3}{4}\left(\frac{c^{\prime}}{c}\right)^{2}-\frac{b}{2} \frac{c^{\prime}}{c}+\frac{1}{2} \frac{c^{\prime \prime}}{c} .
$$

From (1.2) we see that a solution $w(z)$ of (1.1) is meromorphic if and only if the corresponding solution $u(z)$ of $(1.3)$ is meromorphic. So the numbers of meromorphic solutions of (1.1) and (1.3) are the same.

In [1, 6, 12, 13, the maximum number of distinct meromorphic solutions of (1.3) has been discussed. The main results are summarized in the following three theorems.

TheOREM A ([1, Proposition 2.1]). If the Riccati differential equation (1.3) with $A(z)$ meromorphic has at least three distinct meromorphic solutions $u_{1}(z), u_{2}(z), u_{3}(z)$, then this equation has a one-parameter family $\left\{y_{c}(z): c \in \mathbb{C}\right\}$ of distinct meromorphic solutions with the property that any meromorphic solution $u(z) \not \equiv u_{1}(z)$ of (1.3) satisfies $u(z)=y_{c}(z)$ for some $c \in \mathbb{C}$.

THEOREM B. Suppose that $A(z)$ is meromorphic and has at least one pole.

(i) If $A(z)$ has at least one simple pole, then (1.3) admits at most one meromorphic solution (Proposition 9.1.6 in [6]).

(ii) If $A(z)$ has exactly one simple pole and no poles of higher multiplicity, then (1.3) admits exactly one meromorphic solution (Example 6.2 in [1]).

(iii) If $A(z)$ has at least one pole of odd multiplicity $m \geq 3$, then (1.3) admits no meromorphic solutions. If all poles of $A(z)$ with multiplicity $m \geq 3$ are of even multiplicity, then (1.3) admits at most two distinct meromorphic solutions (Theorem 6.12 in [1]).

(iv) If $A(z)$ has a double pole such that $4 c_{-2} \notin E$, then (1.3) admits at most two distinct meromorphic solutions. Moreover, if $4 c_{-2}=1$, then (1.3) admits at most one meromorphic solution (Theorem 6.4 in [1]).

In Theorem B, $c_{-2}$ denotes the coefficient of the first term of the Laurent expansion of $A(z)$ at a double pole $z_{0}$, i.e., 


$$
A(z)=\frac{c_{-2}}{\left(z-z_{0}\right)^{2}}+\cdots,
$$

and

$$
E:=\left\{1-n^{2} \mid n \text { is an integer } \geq 2\right\} .
$$

Theorem C ([12, Theorem 5]). Suppose that $A(z)$ is a transcendental meromorphic function of finite order. If $\delta(\infty, A)>0$, then (1.3) admits at most two distinct meromorphic solutions of finite order.

Some results on the growth of meromorphic solutions of Riccati differential equations have been obtained in [5, 6, 9]. The main results claimed are as follows.

TheOREM D ([9]). Suppose that $A(z)$ is a rational function that can be represented as $A(z)=a z^{\gamma}+\cdots$ in the neighborhood of infinity. If $u(z)$ is a transcendental meromorphic solution of (1.3), then $\sigma(u)=1+\gamma / 2$. Furthermore, if $\gamma<-1$, then (1.3) admits no transcendental meromorphic solutions.

Theorem E ([5, Theorem 4.6.3]). Suppose that the Riccati differential equation

$$
w^{\prime}=a(z)+b(z) w+w^{2}
$$

has rational coefficients, and the limits $\lim _{z \rightarrow \infty} a(z)|z|^{-\alpha}$ and $\lim _{z \rightarrow \infty} b(z)|z|^{-\beta}$ are finite non-zero constants. If $w(z)$ is a meromorphic solution of (1.4) with infinitely many poles, then $\sigma(w)=1+\max \{\beta, \alpha / 2\}$.

However the following example shows that Theorem $\mathrm{E}$ is not always true.

EXAmPLE 1. The function $w(z)=\tan z-z^{2}$ satisfies the equation

$$
w^{\prime}=z^{4}-2 z+1+2 z^{2} w+w^{2},
$$

where $a(z)=z^{4}-2 z+1, b(z)=2 z^{2}, \alpha=4, \beta=2$. Obviously, $\sigma(w)=$ 1 , while by Theorem $\mathrm{E}$, we get $\sigma(w)=1+\max \{2,4 / 2\}=3$. This is a contradiction.

In this paper, we will investigate the equation (1.4) and correct Theorem E. Our method is totally different from the method used in [5].

TheOREM 1.1. Suppose that $a(z)$ and $b(z)$ are two rational functions such that as $z \rightarrow \infty$,

$$
\begin{aligned}
& |a(z)| \sim c_{1}|z|^{m}, \quad|b(z)| \sim c_{2}|z|^{n}, \\
& \left|a(z)-\frac{b^{2}(z)}{4}+\frac{b^{\prime}(z)}{2}\right| \sim c_{3}|z|^{k},
\end{aligned}
$$

where $c_{1}, c_{2}, c_{3}$ are three constants with $c_{1} c_{2} c_{3} \neq 0$, and $m, n, k$ are three integers. 
(i) Every transcendental meromorphic solution $w(z)$ of (1.4) satisfies

$$
\sigma(w)=1+\min \{k / 2, \max \{n, m / 2\}\} \geq 1 / 2 .
$$

(ii) If $k \geq-1$, then (1.4) admits at most two distinct rational solutions.

REMARK 1. By a brief inspection of the proof of Theorem 1.1, we see that if $a(z)-b^{2}(z) / 4+b^{\prime}(z) / 2 \equiv 0$, then all the meromorphic solutions of (1.4) are $w(z)=-\frac{1}{2} b(z)$ and $y_{c}(z)=-\frac{1}{z+c}-\frac{1}{2} b(z)(c \in \mathbb{C})$. So (1.4) admits no transcendental meromorphic solutions in this situation.

From Theorem 1.1, we can easily get the following corollary.

COROLlary 1.1. Suppose that $a(z)$ and $b(z)$ are two rational functions such that $|a(z)| \sim c_{1}|z|^{m}$ and $|b(z)| \sim c_{2}|z|^{n}$ as $z \rightarrow \infty$, where $c_{1}, c_{2}$ are constants with $c_{1} c_{2} \neq 0$, and $m, n$ are integers. If $2 n \neq m$, then every transcendental meromorphic solution $w(z)$ of (1.4) satisfies

$$
\sigma(w)=1+\max \{n, m / 2\} \geq 1 / 2 .
$$

Remark 2. Example 1 shows that the condition $m \neq 2 n$ in Corollary 1.1 cannot be omitted. In the case of $m=2 n$, we need the additional condition (1.6) to get (1.7).

In the case where at least one of $a(z)$ and $b(z)$ is transcendental, we get

TheOREM 1.2. Suppose that $a(z)$ is a meromorphic function and the number of multiple poles of $a(z)$ is finite. Suppose that $b(z)$ is a meromorphic function with finitely many poles. Suppose further that $\sigma=\max \{\sigma(a), \sigma(b)\}$ $<\infty$.

(i) Every meromorphic solution $w(z)$ of (1.4) satisfies $\sigma_{2}(w)=\lambda_{2}(1 / w)$ $\leq \sigma$.

(ii) If $\sigma(a)>\sigma(b)$, then (1.4) admits at most two distinct meromorphic solutions of finite order.

REMARK 3. If $b(z) \equiv 0$, then (1.4) becomes (1.3) with $A(z)=a(z)$. In this case, the conclusions in Theorems 1.1 and 1.2 also hold.

In the above theorems we deal with the growth order. Next we will consider the problem of Borel exceptional values. It is well-known that a meromorphic function of finite order admits at most two Borel exceptional values. We will show in Theorem 1.3 that every meromorphic solution $w(z)$ of (1.1) admits at most one Borel exceptional value under certain assumptions. From transformation (1.2) and Theorem D, we know that if the coefficients of (1.1) are rational, then $\max \{\sigma(a), \sigma(b), \sigma(c)\}=0<\sigma(w)<\infty$ for every transcendental meromorphic solution $w(z)$ of (1.1).

TheOREM 1.3. Suppose that $a(z), b(z), c(z)$ are meromorphic functions of finite order, and at least two of them are linearly independent. Suppose 
that $w(z)$ is a meromorphic solution of (1.1) with $\max \{\sigma(a), \sigma(b), \sigma(c)\}=$ $\beta<\sigma(w)<\infty$. Then $w(z)$ admits at most one Borel exceptional value.

COROLlary 1.2. Suppose that $a(z)$ and $b(z)$ are two rational functions and at least one of them is non-constant. If $w(z)$ is a transcendental meromorphic solution of (1.4), then $w(z)$ admits at most one Borel exceptional value.

REMARK 4. By transformation (1.2), equation (1.1) can be transformed into (1.3). From (1.2), we see that $w(z)$ and $u(z)$ may have different growth orders and different numbers of Borel exceptional values. See the following two examples.

EXAMPLE 2. The meromorphic function $w(z)=1 / z-e^{z}$ satisfies the equation

$$
w^{\prime}=-\frac{2}{z^{2}}+e^{2 z}-e^{z}+2 e^{z} w+w^{2} .
$$

By $(1.2), u(z)=1 / z$ satisfies the equation

$$
u^{\prime}=-2 / z^{2}+u^{2} .
$$

Obviously, $\sigma(u)=0, \sigma(w)=1$.

EXAmple 3. The meromorphic function $w(z)=-1 / z+\tan z$ satisfies the equation

$$
w^{\prime}=\frac{2}{z^{2}}+1+\frac{2}{z} w+w^{2} .
$$

By (1.2), $u(z)=\tan z$ satisfies the equation

$$
u^{\prime}=1+u^{2} \text {. }
$$

The solution $w(z)$ has no Borel exceptional value, while $u(z)$ has two Borel exceptional values, namely $i$ and $-i$.

\section{Lemmas for the proofs of theorems}

Lemma 2.1 ([7, p. 193]). Let $g(z)$ be a meromorphic function. If all poles of $g(z)$ are simple and the residues at all poles are integers, then $\exp \left\{\int^{z} g(t) d t\right\}$ is meromorphic. In particular, if all poles of $g(z)$ are simple and the residues at all poles are positive integers, then $\exp \left\{\int^{z} g(t) d t\right\}$ is entire.

Lemma $2.2([2$, Lemma 1]). Suppose that $w(z)$ is a meromorphic function with $\sigma(w)=\beta<\infty$. Then for any given $\varepsilon>0$, there is a set $E \subset(1, \infty)$ with finite linear measure and finite logarithmic measure such that

$$
|w(z)| \leq \exp \left\{r^{\beta+\varepsilon}\right\}
$$

for $|z|=r \notin[0,1] \cup E, r \rightarrow \infty$. 
Lemma 2.3 ([3, pp. 69-70] or [10, p. 82]). Suppose that $f_{1}(z), \ldots, f_{n}(z)$ are meromorphic functions and $g_{1}(z), \ldots, g_{n}(z)$ are entire functions satisfying the following conditions:

(1) $\sum_{j=1}^{n} f_{j}(z) e^{g_{j}(z)} \equiv 0$;

(2) $g_{j}(z)-g_{k}(z)$ are not constants for $1 \leq j<k \leq n$;

(3) for $1 \leq j \leq n, 1 \leq h<k \leq n$,

$$
T\left(r, f_{j}\right)=o\left\{T\left(r, e^{g_{h}-g_{k}}\right)\right\} \quad \text { n.e. as } r \rightarrow \infty .
$$

Then $f_{j}(z) \equiv 0(j=1, \ldots, n)$.

\section{Proofs of Theorems 1.1 and 1.2 and examples}

Proof of Theorem 1.1. By the transformation

$$
w(z)=u(z)-\frac{1}{2} b(z)
$$

the equation (1.4) can be transformed into the equation (1.3) with

$$
A(z)=a(z)-\frac{b^{2}(z)}{4}+\frac{b^{\prime}(z)}{2} .
$$

(a) We first prove the conclusion (i).

Since $w(z)$ is a transcendental meromorphic solution of $(1.4)$ and $b(z)$ is a rational function, by (3.1) we see that $u(z)=w(z)+\frac{1}{2} b(z)$ is a transcendental meromorphic solution of (1.3). By (1.3) we know that outside the poles of $A(z), u(z)$ has at most simple poles with residue -1 . Since $A(z)$ is rational, $A(z)$ can have only finitely many poles; denote them by $z_{1}, \ldots, z_{s}$. By (1.3) every $z_{j}(j \in\{1, \ldots, s\})$ is a pole of $u(z)$. Let the multiplicity of $u(z)$ at $z_{j}$ be $m_{j}$. Then $u(z)$ can be represented in the form

$$
\begin{aligned}
u(z)= & \frac{c_{m_{1}}^{1}}{\left(z-z_{1}\right)^{m_{1}}}+\cdots+\frac{c_{1}^{1}}{z-z_{1}}+\cdots+\frac{c_{m_{j}}^{j}}{\left(z-z_{j}\right)^{m_{j}}}+\cdots+\frac{c_{1}^{j}}{z-z_{j}} \\
& +\cdots+\frac{c_{m_{s}}^{s}}{\left(z-z_{s}\right)^{m_{s}}}+\cdots+\frac{c_{1}^{s}}{z-z_{s}}+\varphi(z),
\end{aligned}
$$

where $\varphi(z)$ is analytic at the points $z_{1}, \ldots, z_{s}$ and has at most simple poles with residue -1 .

Let

$$
\frac{c_{m_{1}}^{1}}{\left(z-z_{1}\right)^{m_{1}}}+\cdots+\frac{c_{1}^{1}}{z-z_{1}}+\cdots+\frac{c_{m_{s}}^{s}}{\left(z-z_{s}\right)^{m_{s}}}+\cdots+\frac{c_{1}^{s}}{z-z_{s}}=\frac{Q(z)}{P(z)},
$$

where $P(z), Q(z)$ are relatively prime polynomials with $\operatorname{deg} Q<\operatorname{deg} P$ or $Q(z) \equiv 0$. Then

$$
u(z)=\frac{Q(z)}{P(z)}+\varphi(z) .
$$


Since $u(z)$ is transcendental, it follows that $\varphi(z)=u(z)-Q(z) / P(z) \not \equiv 0$ and $\varphi(z)$ is transcendental. By Lemma 2.1, there exists an entire function $g(z)=\exp \left\{-\int^{z} \varphi(t) d t\right\}$ such that $\varphi(z)=-g^{\prime}(z) / g(z)$. So

$$
u(z)=\frac{Q(z)}{P(z)}-\frac{g^{\prime}(z)}{g(z)} .
$$

Since $u(z)$ is transcendental, so is $g(z)$. Substituting (3.3) into (1.3), we have

$$
\frac{g^{\prime \prime}}{g}-\frac{2 Q}{P} \frac{g^{\prime}}{g}+A(z)+\frac{Q^{2}}{P^{2}}-\frac{Q^{\prime}}{P}+\frac{Q P^{\prime}}{P^{2}}=0 .
$$

Since $g(z)$ is a transcendental entire function, from (3.4), $\operatorname{deg} Q<\operatorname{deg} P$ or $Q(z) \equiv 0$, and Wiman-Valiron theory ([8]), we have

$$
k \geq-1
$$

and

$$
\sigma(g)=\frac{k+2}{2} .
$$

We divide our discussion into two cases.

Case $1: k$ is odd. Since $(k+2) / 2$ is not an integer, by Hadamard's factorization theory and (3.6), we get $\lambda(g)=\sigma(g)=(k+2) / 2$.

CASE 2: $k$ is even. Let $g(z)=H(z) e^{P_{1}(z)}$, where $H(z)$ is the canonical product of the zeros of $g(z)$. Since $\sigma(g)=(k+2) / 2$, we see that $P_{1}(z)$ is a polynomial of degree at most $(k+2) / 2$. Since $u(z)=Q / P-g^{\prime} / g=$ $Q / P-H^{\prime} / H-P_{1}^{\prime}$ is transcendental, it follows that $H(z)$ is transcendental. Substituting $g^{\prime} / g=H^{\prime} / H+P_{1}^{\prime}$ into (3.4), we get

$$
\frac{H^{\prime \prime}}{H}+\left(2 P_{1}^{\prime}-\frac{2 Q}{P}\right) \frac{H^{\prime}}{H}+P_{1}^{\prime \prime}+\left(P_{1}^{\prime}\right)^{2}-\frac{2 Q}{P} P_{1}^{\prime}+A(z)+\frac{Q^{2}}{P^{2}}-\frac{Q^{\prime}}{P}+\frac{Q P^{\prime}}{P^{2}}=0
$$

By (3.7) and Wiman-Valiron theory, we get $\sigma(H)=(k+2) / 2$. So $\sigma(H)=$ $\lambda(H)=\lambda(g)=(k+2) / 2$.

Therefore we get $\lambda(g)=(k+2) / 2$ in both cases.

Since $u \cdot u=u^{\prime}-A(z)$, by the Tumura-Clunie Lemma we get

$$
m(r, u)=S(r, u)+O(m(r, A)),
$$

where $S(r, u)=O(\log T(r, u)+\log r)$ n.e. as $r \rightarrow \infty$. Since $A(z)$ is rational, we have $m(r, A)=O(\log r)$. So

$$
T(r, u)=O(\log T(r, u)+\log r)+N(r, u) \text { n.e. as } r \rightarrow \infty .
$$

This gives $\sigma(u) \leq \lambda(1 / u)$. As obviously, $\lambda(1 / u) \leq \sigma(u)$ we obtain $\sigma(u)=$ $\lambda(1 / u)$.

Since $\varphi(z)=-g^{\prime}(z) / g(z)$ and the residue of $\varphi(z)$ at every pole is -1 , we see that every zero of $g(z)$ is simple. So $\bar{\lambda}(g)=\lambda(g)=(k+2) / 2$. By 
(3.3), we get $\lambda(1 / u)=\bar{\lambda}(g)$. Therefore $\sigma(u)=\lambda(1 / u)=\bar{\lambda}(g)=(k+2) / 2$. Combining (3.1) and (3.5), we get

$$
\sigma(w)=\sigma(u)=\frac{k+2}{2} \geq \frac{1}{2} .
$$

If $k \leq \max \{2 n, m\}$, then $k / 2 \leq \max \{n, m / 2\}$. So (3.9) gives

$$
\sigma(w)=1+\min \{k / 2, \max \{n, m / 2\}\} \geq 1 / 2 .
$$

If $k>\max \{2 n, m\}$, then by (1.5) and (1.6) we get $k<-1$. This contradicts (3.5). So we have proved the conclusion (i).

(b) Now we prove the conclusion (ii).

Assume that $u(z)$ is a rational solution of (1.3). Since $A(z) \not \equiv 0$, we have $u(z) \not \equiv 0$. So $u(z)$ can be represented as

$$
u(z)=\frac{Q(z)}{P(z)}+\varphi(z),
$$

where $P(z), Q(z)$ are relatively prime polynomials with $\operatorname{deg} Q<\operatorname{deg} P$ or $Q(z) \equiv 0$, and $\varphi(z)$ is a polynomial. Since $k \geq-1$, it follows that $Q(z) / P(z)$ is not a solution of (1.3). Indeed, if $Q(z) / P(z)$ satisfies (1.3), then

$$
\frac{Q^{\prime} P-Q P^{\prime}}{P^{2}}=A(z)+\frac{Q^{2}}{P^{2}} \text {. }
$$

Since $\operatorname{deg} Q<\operatorname{deg} P$ or $Q(z) \equiv 0$, we have

$$
|A(z)|=\left|\frac{Q^{\prime}}{P}-\frac{Q P^{\prime}}{P^{2}}-\frac{Q^{2}}{P^{2}}\right|=O\left(\frac{1}{|z|^{2}}\right) \quad \text { or } \quad|A(z)| \equiv 0 .
$$

This contradicts $|A(z)| \sim c_{3}|z|^{k}, k \geq-1, c_{3} \neq 0$. So $Q(z) / P(z)$ is not a solution of (1.3). But $u(z)$ is a solution of (1.3). So $\varphi(z)=u(z)-Q(z) / P(z) \not \equiv 0$. Since $\varphi(z)$ is a non-zero polynomial, $g(z)=\exp \left\{-\int^{z} \varphi(t) d t\right\}$ must be a transcendental entire function such that $\varphi(z)=-g^{\prime}(z) / g(z)$. Therefore we have

$$
u(z)=\frac{Q(z)}{P(z)}-\frac{g^{\prime}(z)}{g(z)} .
$$

Now we assume that (1.3) has three distinct rational solutions $u(z)$, $u_{2}(z), u_{3}(z)$. Following the same argument as in (2.1) and (2.2) of [1], we denote

$$
\begin{aligned}
& y_{1}(z)=\frac{1}{u(z)-u_{2}(z)}, \quad y_{2}(z)=\frac{1}{u(z)-u_{3}(z)}, \\
& V_{0}(z)=y_{1}-y_{2}=\frac{u_{2}-u_{3}}{\left(u-u_{2}\right)\left(u-u_{3}\right)} \not \equiv 0 .
\end{aligned}
$$

By calculation, we find that $y_{1}(z), y_{2}(z)$ both satisfy

$$
y^{\prime}+2 u y=1 \text {, }
$$


and $V_{0}(z)$ satisfies

$$
V^{\prime}+2 u V=0 .
$$

So by (3.10) we have

$$
\frac{V_{0}^{\prime}}{V_{0}}=-2 u=-2\left(\frac{Q}{P}-\frac{g^{\prime}}{g}\right) .
$$

This yields

$$
\frac{g^{\prime}}{g}=\frac{1}{2} \frac{V_{0}^{\prime}}{V_{0}}+\frac{Q}{P}
$$

Since we have proved $g(z)$ is transcendentally entire, by Wiman-Valiron theory, we get

$$
\frac{g^{\prime}(z)}{g(z)}=\frac{\nu(r)}{z}(1+o(1)),
$$

where $|z|=r,|g(z)|=M(r, g), r \notin[0,1] \cup E, E \subset(1, \infty)$ is of finite logarithmic measure, and $\nu(r)$ denotes the central index of $g(z)$. Substituting (3.12) into (3.11), we have

$$
\frac{\nu(r)}{z}(1+o(1))=\frac{1}{2} \frac{V_{0}^{\prime}}{V_{0}}+\frac{Q}{P} .
$$

Since $Q(z), P(z)$ are polynomials satisfying $\operatorname{deg} Q<\operatorname{deg} P$ or $Q(z) \equiv 0$, and $V_{0}$ is rational, by (3.13), when $r \notin[0,1] \cup E$ and $r$ is sufficiently large, we have

$$
\nu(r) \leq 2 r\left|\frac{1}{2} \frac{V_{0}^{\prime}}{V_{0}}\right|+2 r\left|\frac{Q}{P}\right| \leq M,
$$

where $M$ is a positive constant. (3.14) contradicts the fact that $g(z)$ is transcendentally entire.

Hence (1.3) admits at most two rational solutions. Since $b(z)$ is rational, by (3.1) we see that (1.4) admits at most two rational solutions. This finishes the proof.

Remark 5. The conclusion (i) in Theorem 1.1 can also be proved by use of Theorem D. However, the proof of Theorem D in [9] is complicated, while using our method of proof we can give a brief proof of Theorem D.

EXAmple 4. Consider the equation

$$
w^{\prime}=\frac{-2 z-3}{4 z^{2}}-\frac{5}{4}+z^{2}+2 z w+w^{2},
$$

where $a(z)=\frac{-2 z-3}{4 z^{2}}-\frac{5}{4}+z^{2}, b(z)=2 z, m=2, n=1, k=0$. 
The equation (3.15) admits meromorphic solutions

$$
\begin{aligned}
& w_{1}(z)=\frac{1}{2 z}+\frac{1}{2}-z, \quad w_{2}(z)=\frac{1}{2 z}+\frac{1}{2}-\frac{z}{z-1}-z, \\
& y_{c}(z)=\frac{1}{2 z}+\frac{1}{2}-z-\frac{z e^{z}}{(z-1) e^{z}+c} \quad(c \neq 0, c \in \mathbb{C}) .
\end{aligned}
$$

By (3.1), (3.2) and Theorem A, (3.15) has no other meromorphic solutions. Obviously, $w_{1}(z)$ and $w_{2}(z)$ are rational functions, and for every $c(c \neq$ $0, c \in \mathbb{C}), y_{c}(z)$ is a transcendental meromorphic function with $\sigma\left(y_{c}\right)=1=$ $1+\min \{k / 2, \max \{n, m / 2\}\} \geq 1 / 2$.

Example 4 illustrates Theorem 1.1(i) in the case $k<\max \{2 n, m\}$. This example also shows that Theorem 1.1(ii) is best possible in the sense that two rational solutions may appear.

EXAMPLE 5. All the meromorphic solutions of the equation

are

$$
w^{\prime}=\frac{2}{z^{2}}+1+\frac{2}{z} w+w^{2}
$$

$$
w_{1}(z)=-\frac{1}{z}+\tan z
$$

and

$$
y_{c}(z)=\frac{c \tan z-1}{\tan z+c}-\frac{1}{z} \quad(c \in \mathbb{C}),
$$

where $a(z)=2 / z^{2}+1, b(z)=2 / z, m=0, n=-1, k=0$. If $c=i$, then $y_{i}(z)=i-1 / z$; if $c=-i$, then $y_{-i}(z)=-i-1 / z$. Obviously, for every $c(c \in \mathbb{C}, c \neq \pm i), \sigma\left(w_{1}\right)=\sigma\left(y_{c}\right)=1=1+\min \{k / 2, \max \{n, m / 2\}\} \geq 1 / 2$.

Example 5 illustrates Theorem 1.1(i) in the case $k=\max \{2 n, m\}$.

EXAMPLE 6. The equation

$$
w^{\prime}=\frac{1}{144 z^{6}}+\frac{1}{6 z^{3}} w+w^{2}
$$

admits exactly two meromorphic solutions (see Theorem B(iii))

$$
w_{1}(z)=-\frac{1}{z}-\frac{1}{2 z^{2}}-\frac{1}{12 z^{3}} \quad \text { and } \quad w_{2}(z)=-\frac{1}{z}+\frac{1}{2 z^{2}}-\frac{1}{12 z^{3}},
$$

where $a(z)=1 / 144 z^{6}, b(z)=1 / 6 z^{3}, m=-6, n=-3, k=-4, k>$ $\max \{2 n, m\}$. Obviously, $w_{1}(z)$ and $w_{2}(z)$ are rational functions.

Example 6 illustrates the case $k>\max \{2 n, m\}$. From the proof of Theorem 1.1(i), we see that if $k>\max \{2 n, m\}$, then (1.4) admits no transcendental meromorphic solutions.

EXAmple 7 . The function $w(z)=-1 / z-z$ satisfies the equation

$$
w^{\prime}=-\frac{2}{z}-z^{2}-2 z-3-2 w+w^{2},
$$


where $a(z)=-2 / z-z^{2}-2 z-3, b(z)=-2, m=2, n=0, k=2$. By (3.1), (3.2) and Theorem $\mathrm{B}(\mathrm{i}), w(z)$ is the only meromorphic solution of this equation. Obviously, $w(z)$ is a rational function.

Example 7 shows that under the conditions of Theorem 1.1(ii), the equation (1.4) may have only one rational solution.

EXAMPLE 8. All the meromorphic solutions of the equation

$$
w^{\prime}=-\frac{3}{4 z^{2}}+z^{2}-1+2 z w+w^{2}
$$

are

$$
w_{1}(z)=-\frac{3}{2 z}-z
$$

and

$$
y_{c}(z)=-\frac{3}{2 z}-z-\frac{1}{c z^{3}-z / 2} \quad(c \in \mathbb{C}),
$$

where $a(z)=-3 / 4 z^{2}+z^{2}-1, b(z)=2 z, m=2, n=1, k=-2$.

Example 8 shows that if $k<-1$, then the equation (1.4) may have infinitely many rational solutions.

Proof of Theorem 1.2. (a) Since $w(z)$ is a meromorphic solution of (1.4), we see that outside the poles of $b(z)$ and the multiple poles of $a(z), w(z)$ has at most simple poles with residue -1 . By the hypotheses of $a(z)$ and $b(z)$, using the same method of proof as is used in Theorem 1.1(i), we find that there exist two relatively prime polynomials $P(z)$ and $Q(z)$ with $\operatorname{deg} Q<$ $\operatorname{deg} P$ or $Q(z) \equiv 0$ such that $w(z)-Q(z) / P(z)$ has at most simple poles with residue -1 . By Lemma 2.1, there exists an entire function $g(z)=$ $\exp \left\{-\int^{z}(w(t)-Q(t) / P(t)) d t\right\}$, such that

$$
w(z)-\frac{Q(z)}{P(z)}=-\frac{g^{\prime}(z)}{g(z)} .
$$

If $g(z)$ is a polynomial, then by (3.16) we see that $w(z)$ is rational. So we get conclusion (i). If $g(z)$ is a transcendental entire function, then by Wiman-Valiron theory, we get

$$
\frac{g^{(j)}(z)}{g(z)}=\left(\frac{\nu(r)}{z}\right)^{j}(1+o(1)) \quad(j=1,2),
$$

where $|z|=r,|g(z)|=M(r, g), r \notin[0,1] \cup E, E \subset(1, \infty)$ is of finite logarithmic measure, and $\nu(r)$ denotes the central index of $g(z)$.

Substituting (3.16) into (1.4), we get

$$
\frac{g^{\prime \prime}}{g}-\left(b+\frac{2 Q}{P}\right) \frac{g^{\prime}}{g}+a+b \frac{Q}{P}+\frac{Q^{2}}{P^{2}}-\frac{Q^{\prime}}{P}+\frac{Q P^{\prime}}{P^{2}}=0 .
$$


Substituting (3.17) into (3.18), we get

$$
\begin{aligned}
& \frac{\nu^{2}(r)}{z^{2}}(1+o(1))-\left(b+\frac{2 Q}{P}\right) \frac{\nu(r)}{z} \\
& (1+o(1)) \\
& +a+b \frac{Q}{P}+\frac{Q^{2}}{P^{2}}-\frac{Q^{\prime}}{P}+\frac{Q P^{\prime}}{P^{2}}=0 .
\end{aligned}
$$

By (3.19), when $r \notin[0,1] \cup E$ and $r$ is sufficiently large, we have

$$
\nu^{2}(r) \leq 2 r^{2}\left|\left(b+\frac{2 Q}{P}\right) \frac{\nu(r)}{z}(1+o(1))\right|+2 r^{2}\left|a+b \frac{Q}{P}+\frac{Q^{2}}{P^{2}}-\frac{Q^{\prime}}{P}+\frac{Q P^{\prime}}{P^{2}}\right|
$$

Since $g(z)$ is transcendentally entire, we have $\nu(r) \rightarrow \infty$ (as $r \rightarrow \infty$ ). So by (3.20), when $r \notin[0,1] \cup E$ and $r$ is sufficiently large, we get

$$
\nu(r) \leq 4 r\left|b+\frac{2 Q}{P}\right|+2 r^{2}\left|a+b \frac{Q}{P}+\frac{Q^{2}}{P^{2}}-\frac{Q^{\prime}}{P}+\frac{Q P^{\prime}}{P^{2}}\right| .
$$

Since $\sigma=\max \{\sigma(a), \sigma(b)\}<\infty$, by Lemma 2.2, for any given $\varepsilon>0$, there is a set $E_{1} \subset(1, \infty)$ with finite logarithmic measure such that

$$
|a(z)| \leq \exp \left\{r^{\sigma+\varepsilon}\right\}, \quad|b(z)| \leq \exp \left\{r^{\sigma+\varepsilon}\right\}
$$

for $|z|=r \notin[0,1] \cup E_{1}, r \rightarrow \infty$. Since $P(z), Q(z)$ are polynomials with $\operatorname{deg} Q<\operatorname{deg} P$ or $Q(z) \equiv 0$, by (3.21), (3.22), when $r \notin[0,1] \cup E \cup E_{1}$ and $r$ is sufficiently large, we have

$$
\nu(r) \leq \exp \left\{r^{\sigma+2 \varepsilon}\right\} .
$$

This gives $\sigma_{2}(g) \leq \sigma+2 \varepsilon$. By the arbitrariness of $\varepsilon$, we get $\sigma_{2}(g) \leq \sigma$.

Since $w \cdot w=w^{\prime}-a(z)-b(z) w$, by the Tumura-Clunie Lemma we have

$$
m(r, w)=O(m(r, a)+m(r, b))+S(r, w),
$$

where $S(r, w)=O(\log T(r, w)+\log r)$ n.e. as $r \rightarrow \infty$. So

$$
T(r, w)=O(\log T(r, w)+\log r+m(r, a)+m(r, b))+N(r, w) \text { n.e. }
$$

as $r \rightarrow \infty$.

Since $a(z)$ and $b(z)$ are of finite order, by (3.24), we get $\sigma_{2}(w) \leq \lambda_{2}(1 / w)$. In addition, we have $\sigma_{2}(w) \geq \lambda_{2}(1 / w)$. So $\sigma_{2}(w)=\lambda_{2}(1 / w)$.

Hence from $\sigma_{2}(g) \leq \sigma$ and $(3.16)$, we get $\sigma_{2}(w)=\lambda_{2}(1 / w) \leq \sigma_{2}(g) \leq \sigma$. So conclusion (i) holds.

(b) By the transformation

$$
w(z)=u(z)-\frac{1}{2} b(z)
$$

the equation (1.4) can be transformed into (1.3) with

$$
A(z)=a(z)-\frac{b^{2}(z)}{4}+\frac{b^{\prime}(z)}{2} .
$$


If $A(z)$ has at least one simple pole, then by Theorem B(i), (1.3) admits at most one meromorphic solution. So by (3.25), (1.4) admits at most one meromorphic solution. If $A(z)$ has no simple poles, then by (3.26) and the hypotheses on $a(z)$ and $b(z), A(z)$ has only finitely many poles. So $N(r, A)=$ $O(\log r)$. Since $\sigma(a)>\sigma(b), A(z)$ must be a transcendental meromorphic function. So $\delta(\infty, A)=1>0$. By Theorem $\mathrm{C}$, the equation (1.3) admits at most two distinct meromorphic solutions of finite order. By (3.25) and $\sigma(b)<\infty$, we get conclusion (ii).

We give three examples to illustrate Theorem 1.2.

EXAMPLE 9. All the meromorphic solutions of the equation

$$
w^{\prime}=e^{2 z}+w+w^{2}
$$

are

$$
w_{1}(z)=e^{z} \tan e^{z}
$$

and

$$
y_{c}(z)=\frac{\left(c e^{z}+\frac{1}{2}\right)\left(\tan e^{z}+c\right)-\left(c^{2}+1\right) e^{z}}{\tan e^{z}+c}-\frac{1}{2} \quad(c \in \mathbb{C}),
$$

where $a(z)=e^{2 z}, b(z)=1$. If $c=i$, then $y_{i}(z)=i e^{z} ;$ if $c=-i$, then $y_{-i}(z)=$ $-i e^{z}$. Obviously, $\lambda_{2}\left(1 / w_{1}\right)=\sigma_{2}\left(w_{1}\right)=1 \leq \max \{\sigma(a), \sigma(b)\}, \lambda_{2}\left(1 / y_{ \pm i}\right)=$ $\sigma_{2}\left(y_{ \pm i}\right)=0 \leq \max \{\sigma(a), \sigma(b)\}$ and for every $c(c \in \mathbb{C}, c \neq \pm i), \lambda_{2}\left(1 / y_{c}\right)=$ $\sigma_{2}\left(y_{c}\right)=1 \leq \max \{\sigma(a), \sigma(b)\}$.

Example 9 illustrates Theorem 1.2(i). This example also shows that Theorem 1.2(ii) is best possible in the sense that two meromorphic solutions of finite order may appear.

EXAmple 10. The function $w(z)=e^{z}$ satisfies the equation

$$
w^{\prime}=(2 / z) e^{z}+e^{z}-e^{2 z}-(2 / z) w+w^{2},
$$

where $a(z)=(2 / z) e^{z}+e^{z}-e^{2 z}, b(z)=-2 / z$. By (3.25), (3.26) and Theorem $\mathrm{B}(\mathrm{i}), w(z)$ is the only meromorphic solution of this equation. Obviously, $\sigma(w)=1<\infty$.

Example 10 shows that under the conditions of Theorem 1.2(ii), the equation (1.4) may have only one meromorphic solution with finite order.

EXAMPLE 11. All the meromorphic solutions of the equation

$$
w^{\prime}=e^{2 z}-e^{z}+1+2 e^{z} w+w^{2}
$$

are

$$
w_{1}(z)=\tan z-e^{z}
$$

and

$$
y_{c}(z)=\frac{c \tan z-1}{\tan z+c}-e^{z} \quad(c \in \mathbb{C}),
$$


where $a(z)=e^{2 z}-e^{z}+1, b(z)=2 e^{z}$. Obviously all the meromorphic solutions of this equation are of finite order.

Example 11 shows that if $\sigma(a) \leq \sigma(b)$, then the equation (1.4) may have infinitely many solutions with finite order.

\section{Proofs of Theorem 1.3 and Corollary 1.2 and examples}

Proof of Theorem 1.3. Assume that $w(z)$ has two Borel exceptional values $a_{1}, b_{1}\left(a_{1} \neq b_{1}\right)$. We divide our discussion into two cases.

CASE (i): $a_{1} \neq \infty, b_{1} \neq \infty$. Let

$$
F(z)=\frac{w(z)-a_{1}}{w(z)-b_{1}}
$$

So $F(z)$ has two Borel exceptional values $0, \infty, \lambda(F)<\sigma(F), \lambda(1 / F)<$ $\sigma(F)$ and $\sigma(F)=\sigma(w)$. By Hadamard's factorization theory, $F(z)$ can be represented as $F(z)=\left(H_{1}(z) / H_{2}(z)\right) e^{P(z)}$, where $H_{1}(z)$ and $H_{2}(z)$ denote respectively the canonical products of zeros and poles of $F(z)$. We have

$$
\sigma\left(H_{1}\right)=\lambda\left(H_{1}\right)=\lambda(F)<\sigma(F), \quad \sigma\left(H_{2}\right)=\lambda\left(H_{2}\right)=\lambda\left(\frac{1}{F}\right)<\sigma(F) .
$$

Since $\beta<\sigma(F)=\sigma(w)<\infty, P(z)$ is a polynomial of degree $n(\geq 1)$ and $\sigma(F)=n$.

From

$$
F(z)=\frac{w(z)-a_{1}}{w(z)-b_{1}}=\frac{H_{1}(z)}{H_{2}(z)} e^{P(z)}
$$

we get

$$
w=\frac{a_{1} H_{2}-b_{1} H_{1} e^{P}}{H_{2}-H_{1} e^{P}} .
$$

Substituting (4.1) into (1.1), we get

$$
\begin{aligned}
0= & \left(a(z)+b(z) b_{1}+c(z) b_{1}^{2}\right) H_{1}^{2} e^{2 P}+\left[b_{1} P^{\prime} H_{1} H_{2}-2 a(z) H_{1} H_{2}\right. \\
& +b_{1} H_{1}^{\prime} H_{2}-a_{1} H_{1}^{\prime} H_{2}-a_{1} P^{\prime} H_{1} H_{2}-b_{1} H_{1} H_{2}^{\prime}+a_{1} H_{1} H_{2}^{\prime} \\
& \left.-b(z) a_{1} H_{1} H_{2}-b(z) b_{1} H_{1} H_{2}-2 c(z) a_{1} b_{1} H_{1} H_{2}\right] e^{P} \\
& +\left(a(z)+b(z) a_{1}+c(z) a_{1}^{2}\right) H_{2}^{2} .
\end{aligned}
$$

By (4.2) and Lemma 2.3, we get

$$
\left\{\begin{array}{l}
a(z)+b(z) b_{1}+c(z) b_{1}^{2}=0, \\
a(z)+b(z) a_{1}+c(z) a_{1}^{2}=0 .
\end{array}\right.
$$

(4.3) yields $b(z)=-\left(a_{1}+b_{1}\right) c(z), a(z)=a_{1} b_{1} c(z)$. So any two of $a(z), b(z), c(z)$ are dependent, which contradicts our hypotheses.

CASE (ii): One of $a_{1}$ and $b_{1}$ is $\infty$. Without losing generality, let $b_{1}=\infty$, $a_{1} \neq \infty$. 
Let $F(z)=w(z)-a_{1}$. Then $F(z)$ has two Borel exceptional values $0, \infty$, $\lambda(F)<\sigma(F)$ and $\lambda(1 / F)<\sigma(F)$. Following the proof of Case (i), we get a contradiction.

Therefore, $w(z)$ has at most one Borel exceptional value.

Proof of Corollary 1.2. Suppose that $w(z)$ is a transcendental meromorphic solution of (1.4). Since $a(z)$ and $b(z)$ are rational functions, by Theorem 1.1 and Remark 1 , we get $\infty>\sigma(w) \geq 1 / 2>0$. Since $c(z) \equiv 1, a(z)$ and $b(z)$ are rational functions and at least one of them is non-constant, it follows that $\max \{\sigma(a), \sigma(b), \sigma(c)\}=0<\sigma(w)<\infty$ and at least two of $a(z), b(z)$ and $c(z)$ are linearly independent. By Theorem 1.3,w(z) admits at most one Borel exceptional value.

We give several examples to show that our results are best possible in certain senses.

EXAMPLE 12. The function $w(z)=1+\tan z$ satisfies the equation

$$
w^{\prime}=2-2 w+w^{2} .
$$

The solution $w(z)$ has two Borel exceptional values $1+i, 1-i$.

EXAMPLE 13. The function $w(z)=\tan z^{2}+2$ satisfies the equation

$$
w^{\prime}=10 z-8 z w+2 z w^{2} .
$$

The solution $w(z)$ has two Borel exceptional values $2+i, 2-i$.

EXAMPLE 14. The function $u(z)=\frac{1+e^{2 z}}{1-e^{2 z}}$ satisfies the equation

$$
u^{\prime}=-1+u^{2} \text {. }
$$

The solution $u(z)$ has two Borel exceptional values $-1,1$.

From the above three examples, we see that when any two of $a(z), b(z)$ and $c(z)$ are dependent, (1.1) may have a meromorphic solution with two Borel exceptional values. So, the hypothesis of Theorem 1.3 that "at least two of $a(z), b(z)$ and $c(z)$ are linearly independent" cannot be omitted.

EXAMPLE 15. The function $w(z)=e^{z}$ satisfies the equation

$$
w^{\prime}=-e^{z}+w+e^{-z} w^{2},
$$

where $\max \{\sigma(a), \sigma(b), \sigma(c)\}=\sigma(w)=1$. The solution $w(z)$ has two Borel exceptional values $0, \infty$.

EXAMPLE 16. The function $w(z)=e^{z}$ satisfies the equation

$$
w^{\prime}=-e^{e^{z}}+e^{-z} e^{e^{z}} w+e^{-z} w^{2},
$$

where $\max \{\sigma(a), \sigma(b), \sigma(c)\}>\sigma(w)$. The solution $w(z)$ has two Borel exceptional values $0, \infty$.

From Examples 15 and 16, we see that if $\max \{\sigma(a), \sigma(b), \sigma(c)\} \geq \sigma(w)$, then $w(z)$ may have two Borel exceptional values. 
EXAMPLE 17. The function $w(z)=\frac{1}{e^{z}+1 / z}$ satisfies the equation

$$
w^{\prime}=-w+\left(\frac{1}{z}+\frac{1}{z^{2}}\right) w^{2}
$$

The solution $w(z)$ has one Borel exceptional value 0 .

From Examples 3 and 17, we see that under the conditions of Theorem 1.3, the meromorphic solution $w(z)$ of (1.1) may have one or no Borel exceptional value.

Acknowledgements. This project was supported by the National Natural Science Foundation of China (No. 10871076).

\section{References}

[1] S. Bank, G. Gundersen and I. Laine, Meromorphic solutions of the Riccati differential equation, Ann. Acad. Sci. Fenn. Ser. A. I. Math. 6 (1981), 369-398.

[2] Z. X. Chen, The zero, pole and order of meromorphic solutions of differential equations with meromorphic coefficients, Kodai Math. J. 19 (1996), 341-354.

[3] F. Gross, Factorization of Meromorphic Functions, U. S. Government Printing Office, Washington, DC, 1972.

[4] W. K. Hayman, Meromorphic Functions, Clarendon Press, Oxford, 1964.

[5] E. Hille, Ordinary Differential Equations in the Complex Domain, Wiley, New York, 1976.

[6] I. Laine, Nevanlinna Theory and Complex Differential Equations, de Gruyter, Berlin, 1993.

[7] S. Saks and A. Zygmund, Analytic Functions, PWN, Warszawa, 1952.

[8] G. Valiron, Lectures on the General Theory of Integral Functions, Chelsea, New York, 1949.

[9] H. Wittich, Eindeutige Lösungen der Differentialgleichung $w^{\prime}=R(z, w)$, Math. Z. 74 (1960), 278-288.

[10] C. C. Yang and H. X. Yi, Uniqueness Theory of Meromorphic Functions, Kluwer, 2003.

[11] L. Yang, Value Distribution Theory and New Research, Sci. Press, Beijing, 1982.

[12] W. J. Yuan, A note on the Riccati differential equation, J. Math. Anal. Appl. 277 (2003), 367-374.

[13] J. H. Zheng, A note on the Riccati differential equation, ibid. 190 (1995), 285-293.

Ran Ran Zhang, Zong Xuan Chen (corresponding author)

School of Mathematical Sciences

South China Normal University

510631 Guangzhou, People's Republic of China

E-mail: zhrr19820315@163.com

chzx@vip.sina.com

Received 12 October 2009

and in final form 1 July 2010 